\title{
A novel green synthesis and characterization of silver nanoparticles using gum tragacanth and evaluation of their potential catalytic reduction activities with methylene blue and Congo red dyes
}

Murali Krishna Indana, Bhagavanth Reddy Gangapuram, Ramakrishna Dadigala, Rajkumar Bandi and Veerabhadram Guttena*

\begin{abstract}
Background: A facile and eco-friendly method for green synthesis of silver nanoparticles (AgNPs) has been developed using gum tragacanth (GT) (Astragalus gummifer), an abundantly available natural phyto-exudate in India, employing a novel method of ultrasonication process.

Methods: Silver nanoparticles were prepared by the reduction of silver nitrate solution by the aqueous extract of gum tragacanth by ultrasonication method at $45^{\circ} \mathrm{C}$ for about $45 \mathrm{~min}$. The aqueous extract of the gum acts as a reducing as well as stabilizing agent.

Results: The resultant AgNPs were characterized by ultraviolet-visible (UV-Vis) spectroscopy, Fourier transform infrared (FTIR) spectroscopy, transmission electron microscopy (TEM), and X-ray diffraction (XRD) techniques. The influence of gum concentration and silver precursor concentration on the synthesis of AgNPs was studied. The role and effectiveness of nanoparticles in the catalytic reduction of a cationic dye methylene blue (MB), and an anionic azo dye Congo red (CR), were also studied. FTIR analysis revealed that $-\mathrm{OH}$ groups present in the gum matrix might be responsible for the reduction of Ag+ into AgNPs. The X-ray diffraction studies indicated that the resulting AgNPs were highly crystalline with face-centered cubic geometry. TEM studies showed that the average particle size of the synthesized AgNPs was $18 \pm 2 \mathrm{~nm}$.
\end{abstract}

Conclusions: The study highlights the green synthesis of GT-capped AgNPs and the rapid reduction of carcinogenic and toxic contaminants such as MB and CR with the help of GT-capped AgNPs in an eco-friendly manner.

Keywords: Gum tragacanth, Silver nanoparticles, Ultra-sonication, Catalytic reduction, Methylene blue, Congo red

\section{Background}

Metal nanomaterials, in recent times, play a crucial role in a multitude of applications as they were proved to exhibit remarkable improvements in their electronic, physical, chemical, optical, and biological properties when compared to their pure metal counterparts (Thakkar et al. 2010). These colloidal metal nanoparticles possess unique physical properties owing to their extremely

\footnotetext{
* Correspondence: gvbhadram@gmail.com

Department of Chemistry, University College of Science, Osmania University, Hyderabad, Telangana State 500007, India
}

small size and very high surface/volume ratio (Pandey et al. 2016). The extremely small size of these metal nanoparticles offers a very high reactive surface area that contributes to their significant improvement in the properties in comparison to their metal compatriots (Guo and Wang 2007). The extremely small size of nanoparticles, especially in the case of silver nanoparticles (AgNPs), finds their utility in chemical applications such as catalysis, reduction, stabilization, and colorimetric sensors (Murugadoss and Chattopadhyay 2008; Rastogi et al. 2014). The biological applications involving the integration of nanoparticles 
with biological molecules led to the development of therapeutic medicines, diagnostic tools, antimicrobial agents, drug delivery systems, bioimaging tools, labeling agents, therapies in cancer, etc. (Roy et al. 2015, Song and Kim 2009, Kokila et al. 2015, Daniel and Astruc 2004).

The AgNPs are generally synthesized using various methods such as chemical, photochemical, electrochem$i c a l$, laser ablation, $\gamma$-irradiation, and biological conversion (Solomon et al. 2007; Callegari et al. 2003; Pyatenko et al. 2004; Li et al. 2007; Raju et al. 2014). The most popular method by which AgNPs are synthesized is by reducing agents such as sodium borohydride and sodium citrate. Most of these synthetic methods are hazardous since they employ toxic chemicals leading to environmental degradation (Mehndiratta et al. 2013) and biological hazards. Much effort is being focussed, in recent times, by researchers to mitigate the impact of these harmful chemicals' utilization through the integration of "green chemistry principles" for the synthesis of metal nanomaterials using plant extracts (Parveen et al. 2016), biosurfactants, etc. There are many natural biopolymers used for metal nanoparticle synthesis such as gum kondagogu (Rastogi et al. 2014), salmalia malabarica gum (Murali krishna et al. 2015) guar gum (Pandey et al. 2012, Pandey and Mishra 2011, Pandey and Mishra 2013, Pandey et al. 2013, Pandey and Mishra 2014, Pandey and Mishra 2016), chitosan (Wang et al. 2015), gum ghatti (Kora et al. 2012) and cyclodextrin (Maciollek and Ritter 2014). These biopolymers act as stabilizing as well as reducing agents. The characteristics of the metal nanoparticles were further improved when these metal nanoparticles were reacted with natural gums such as gum tragacanth (GT) (Raveendran et al. 2003) due to the increase in the number of active sites, i.e., reactive functional groups on nanoparticles through capping process.

Synthetic dyes are extensively used in a variety of industries such as textiles, paper, polymers, adhesives, ceramics, construction, cosmetics, food, glass, paints, ink, soap, and pharmaceuticals (Zalikha et al. 2012). Dye residues form a group of glaring and grave contaminants since they impart color to the wastewater even at a very low concentration level. The catalytic reduction studies of these dyes assume greater significance in the present context as most of these dyes are known to be toxic when inhaled or ingested orally and pose health hazards such as skin and eye irritation in humans. The adverse effects of these synthetic dyes to the environment include the ability to deplete oxygen in the surface waters and streams thereby affecting the very sustenance of aquatic flora and fauna and causing an inhibitory effect on the photosynthetic activity of plants. The anaerobic degradation products of azo dyes are amines which are very toxic, carcinogenic, and mutagenic in nature. The effluents from industries containing these hazardous dyes need to be removed before they are let out for onland disposal purposes. The treatment technologies such as adsorption, ion exchange (Karcher et al. 2002), filtration, electrode deposition, and chemical precipitation are being widely used to remove the dye contaminants from water. An attempt has been made to alleviate the detrimental effects of these chemicals on the surrounding environment by trying to reduce the dye concentration in the treated effluents such as methylene blue (MB) and Congo red (CR) by employing GT-capped AgNPs as a nanocatalyst. The previous studies on the reduction of $\mathrm{MB}$ and $\mathrm{CR}$ by using gold and silver nanoparticles (Wanyonyi et al. 2014; Ganapuram et al. 2015) as nanocatalysts were also reported.

GT is a naturally occurring complex, an acidic polysaccharide obtained from the sap of the genus Astragalus, including $A$. adscendens, A. gummifer, A. brachycalyx, and $A$. tragacanthus, a native genus of Middle Eastern countries and West and South Asia. GT is a highly viscous, odorless, tasteless, water-soluble mixture of highly branched and heterogeneous hydrophilic polysaccharides obtained from the exudate of roots and bark of the plant which can be dried and powdered (Kora and Arunachalam 2012). The molecular weight of a typical gum is reported to be about $8.4 \times$ $10^{5} \mathrm{~g} / \mathrm{mol}$. The biopolymer is a mixture of two polysaccharide fractions viz. water-soluble tragacanthin and water-swellable tragacanthic acid or bassorin. The tragacanthin comprises about $30-40 \%$ in the gum and is a neutral, highly branched, type II arabinogalactan comprising $(1 \rightarrow 6)$ - and $(1 \rightarrow 3)$-linked core chains containing galactose and arabinose units and side groups of $(1 \rightarrow 2)-,(1 \rightarrow 3)$-, and $(1 \rightarrow 5)$-linked arabinose units occurring as a monosaccharide or oligosaccharides. Bassorin, a pectin component, has a chain of $(1 \rightarrow 4)$-linked $\alpha$-Dgalacturonic acid units, some of which are substituted at $\mathrm{O}-3$ with $\beta$-D-xylopyranosyl units and some of these being terminated with D-galactose or L-fucose (Tischer et al. 2002; Hassan et al. 2013; Chenlo et al. 2010). GT is also recognized as a food-grade additive by the Indian Bureau of Standards under Indian Standard IS 7238: 1997.

The present study has been taken up to explore the innumerable potential possibilities that have been foreseen with the green synthesis of AgNPs with GT as a reducing, capping, and stabilizing agent. Also, the attributes of GT which prompted us to get into the study are (i) widespread natural availability, (ii) nontoxic and noncarcinogenic nature, (iii) excellent reducing and stabilizing capability, (iv) compliance with eco-friendly green synthesis principles, and (v) efficient catalytic reduction of MB and CR. The synthesized AgNPs were characterized for ultraviolet-visible (UV-Vis), Fourier transform infrared (FTIR) spectroscopy, X-ray diffraction (XRD), and transmission electron microscopy (TEM) parameters. 


\section{Methods}

\section{Materials}

Silver nitrate $\left(\mathrm{AgNO}_{3}\right), \mathrm{NaBH}_{4}, \mathrm{CR}$, and $\mathrm{MB}$, all of analytical grade, were purchased from E. Merck Limited, Mumbai, India, and GT (99\% purity) was purchased from Loba Chemie, Mumbai, India.

\section{Synthesis of AgNPs using GT}

A $0.5 \%$ (weight/volume $(w / v)$ ) homogeneous gum stock solution was prepared by dissolving $0.5 \mathrm{~g}$ of GT in $100 \mathrm{~mL}$ of deionized water and stirring overnight at room temperature. The solution was then centrifuged at $6000 \mathrm{rpm}$ for $10 \mathrm{~min}$ to separate out any undissolved matter, and the supernatant is used for the experiments to be carried out in the study. A $0.5 \%(w / v)$ solution of $\mathrm{AgNO}_{3}$ is prepared by dissolving $0.5 \mathrm{~g}$ of analytical grade $\mathrm{AgNO}_{3}$ in $100 \mathrm{~mL}$ of deionized water. Then, $5 \mathrm{~mL}$ of gum extract was mixed with $5 \mathrm{~mL}$ of $\mathrm{AgNO}_{3}$ solution in a boiling tube, and this mixture was subjected to ultra sonication at $45{ }^{\circ} \mathrm{C}$ temperature for a 45 -min reaction time (Firdhouse et al. 2012). The conversion of the colorless reaction mixture to the characteristic clear yellow color indicates the formation of AgNPs. A series of samples were prepared by varying the concentration of $\mathrm{AgNO}_{3}(0.1$ to $0.5 \%$ ) while keeping the concentration of GT (0.5 \%) constant. Likewise, the second set of samples were prepared by varying the concentration of GT $(0.1$ to $0.5 \%)$, keeping the concentration of $\mathrm{AgNO}_{3}$ constant $(0.5 \%)$ at the same sonication conditions as mentioned earlier $\left(45^{\circ} \mathrm{C}\right.$ for $\left.45 \mathrm{~min}\right)$.

\section{Characterization of silver nanoparticles}

The UV-Vis spectral analysis of GT-AgNP solution was carried out on a dual-beam UV-Vis spectrophotometer (Shimadzu-3600, Kyoto, Japan). The FTIR spectra of GT alone and GTcapped AgNPs were recorded using FTIR spectrophotometer (model: IRAffinity-1, Shimadzu Corporation, Japan) equipped with attenuated total reflectance (ATR) accessory in the scanning range of 650$4000 \mathrm{~cm}^{-1}$. XRD studies of GT-AgNPs were carried out on X'pert Pro MRD X-ray diffractometer (PANalytical BV, The Netherlands) operating at $40 \mathrm{kV}$ and a current of $30 \mathrm{~mA}$ at a scan rate of $0.388 \mathrm{~min}^{-1}$. TEM analysis was conducted for the morphology and size distribution measurements of the GT-AgNPs using a transmission electron microscope (model: 1200 EX, JEOL Ltd., Japan) operated at an accelerating voltage of $200 \mathrm{kV}$, casting nanoparticle dispersion on carbon-coated copper grids and allowing for drying at room temperature. The particle size distribution was measured by using ImageJ software analysis of TEM micrographs. Zeta potential was determined using dynamic light scattering (Malvern instrument Ltd., Malvern, UK).

\section{Catalytic activity}

$\mathrm{MB}$ and $\mathrm{CR}$ were subjected to reduction using sodium borohydride in the presence of AgNPs in order to assess the efficacy of the catalytic activity of the synthesized AgNPs (Suvith and Philip 2014; Junejo et al. 2014). Freshly prepared $1.0 \mathrm{~mL}$ of $10 \mathrm{mM}$ sodium borohydride solution was mixed with $1.5 \mathrm{~mL}$ of $1 \mathrm{mM} \mathrm{MB}$, and the mixture was made up to $10 \mathrm{~mL}$ using double-distilled water and then stirred for $5 \mathrm{~min}$. Similarly, freshly prepared $1.0 \mathrm{~mL}$ of $10 \mathrm{mM} \mathrm{NaBH}_{4}$ solution was mixed with $1.5 \mathrm{~mL}$ of $1 \mathrm{mM} \mathrm{CR}$, and the solution mixture was made up to $10 \mathrm{~mL}$ using double-distilled water and then stirred for $5 \mathrm{~min}$ as well. Sufficient quantities of synthesized AgNPs were added to both these solutions and mixed for $30 \mathrm{~min}$ with good agitation, and the UV-Vis spectrum of the reaction mixture of $\mathrm{MB}$ was recorded at 1-min intervals of time for a period of $12 \mathrm{~min}$ and a wavelength range of $400-750 \mathrm{~nm}$ at $25{ }^{\circ} \mathrm{C}$ whereas, for $\mathrm{CR}$, the absorption spectrum was recorded at a wavelength range between 300 and $650 \mathrm{~nm}$ with 1-min intervals of time for a period of $15 \mathrm{~min}$ at $25^{\circ} \mathrm{C}$ (Ganapuram et al. 2015). The test was conducted in a standard quartz cuvette of about 3.0-mL volume. The rate constant of the redox reaction was dependent on the variation in absorption band at $664 \mathrm{~nm}$ as a function of time.

\section{Results and discussion \\ UV-Vis spectral analysis}

UV-Vis spectroscopy emerged as one of the most powerful analytical tools for characterization of metal nanoparticles. The synthesized AgNPs were characterized by UV-Vis spectroscopy and observed very well absorption patterns in the UV-Vis spectral analysis. The absorption behavior of AgNPs was attributed to the surface plasmon resonance (SPR), which results from the coherent oscillation of electrons in the conduction band induced by the electromagnetic field, and a characteristic surface plasmon absorption band can be seen in the spectral range of 418-428 $\mathrm{nm}$ (Rai et al. 2009). The role of $\mathrm{AgNO}_{3}$ concentration on the production of AgNPs was studied by UV-Vis spectra of the synthesized AgNPs with different concentrations of $\mathrm{AgNO}_{3}$ (0.1 to $\left.0.5 \%\right)$ with $0.5 \%$ GT for 45 min of ultra sonication at $45{ }^{\circ} \mathrm{C}$ (Fig. 1). It was observed from Fig. 1 that the absorbance intensity of the reaction mixture increased with the increase in $\mathrm{AgNO}_{3}$ concentration which indicates the increase in the efficiency of nanoparticle synthesis. Further, the effect of gum concentration on the synthesis of AgNPs was studied by UV-Vis absorption spectra produced by the ultra sonication of different concentrations of GT $(0.1$ to $0.5 \%)$ with $0.5 \%$ of $\mathrm{AgNO}_{3}$ solution for $45 \mathrm{~min}$ at $45{ }^{\circ} \mathrm{C}$ (Fig. 2). It was noticed that the absorbance intensity of the reaction mixture increased with the 


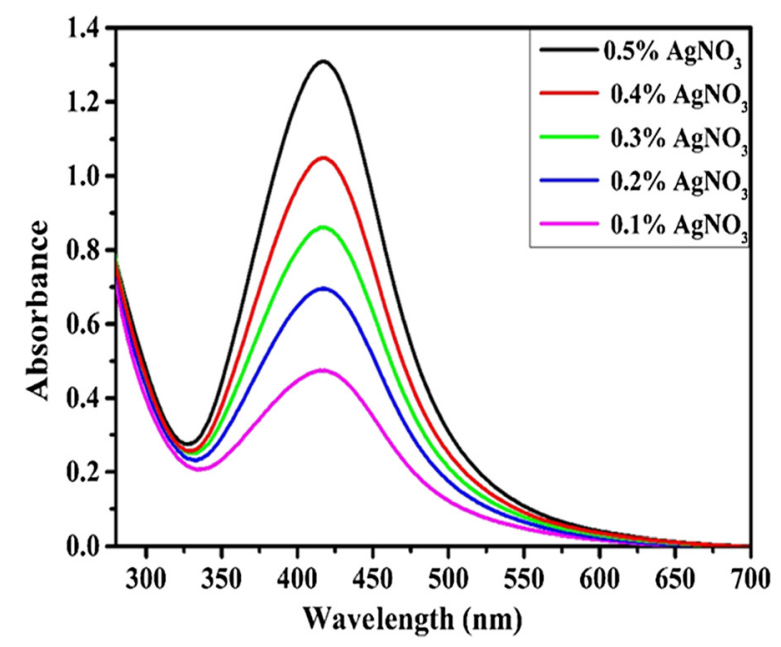

Fig. 1 The UV-Vis absorption spectra of AgNPs synthesized with different concentration of silver nitrate solutions containing $0.5 \%$ of gum tragacanth by ultra sonication for $45 \mathrm{~min}$ at $45^{\circ} \mathrm{C}$

increase in the concentration of GT indicating the higher production rate of nanoparticles.

\section{FTIR analysis}

The stability of synthesized AgNPs was studied in relation to the interaction of various functional groups of GT with $\mathrm{AgNO}_{3}$ solution, and the FTIR spectra were recorded for the aqueous extract of GT and GT-stabilized AgNPs (Fig. 3). The major absorbance peaks for gumstabilized AgNPs were observed at 3442, 2870, 1645, 1380, and $1068 \mathrm{~cm}^{-1}$. The broad band observed at $3442 \mathrm{~cm}^{-1}$ corresponds to the stretching vibration of $\mathrm{O}-\mathrm{H}$ groups in GT. The band at $2870 \mathrm{~cm}^{-1}$ could be attributed to symmetric stretching vibrations of aliphatic

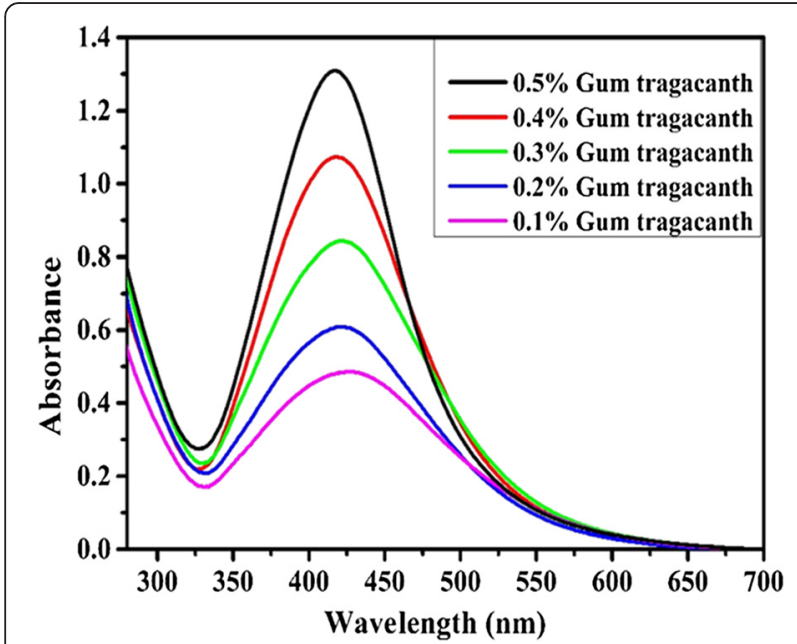

Fig. 2 The UV-Vis absorption spectra of AgNPs synthesized with different concentration of gum tragacanth extracts containing $0.5 \%$ of silver nitrate by ultra sonication for $45 \mathrm{~min}$ at $45^{\circ} \mathrm{C}$

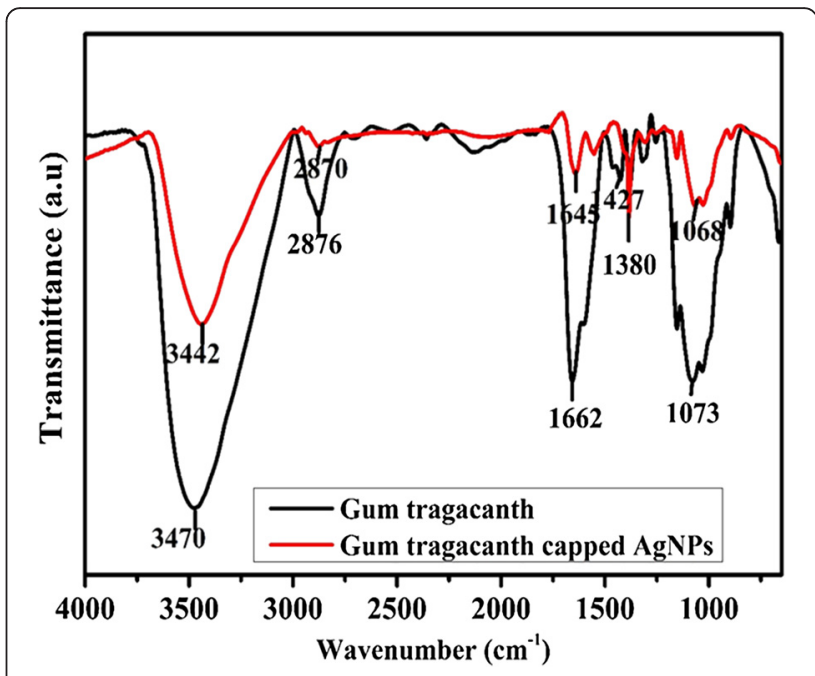

Fig. 3 FTIR spectra of GT aqueous extract (red) and GT-capped AgNPs (black)

$-\mathrm{CH}_{3}$ groups present in the gum. The bands present at 1645 and $1380 \mathrm{~cm}^{-1}$ represent, respectively, the characteristic asymmetrical and symmetrical stretching vibrations of the $-\mathrm{COO}^{-}$group. The absorption band at $1068 \mathrm{~cm}^{-1}$ could be attributed to stretching vibrations of the $\mathrm{C}-\mathrm{O}$ bond in either group. It was clearly observed from the FTIR spectra of both the GT aqueous extract and GT-stabilized AgNPs that the shifting of peaks from 3470 to $3442 \mathrm{~cm}^{-1}, 2876$ to $2870 \mathrm{~cm}^{-1}, 1662$ to $1645 \mathrm{~cm}^{-1}$, and 1073 to $1068 \mathrm{~cm}^{-1}$ with decreased peak intensity suggesting that hydroxyl, methyl, carboxylate, and carbonyl functional groups, respectively, are involved in the formation and stability of AgNPs.

\section{X-ray diffraction}

The crystalline structure of AgNPs stabilized with GT was evidenced using the analytical technique of X-ray diffraction. The recorded XRD spectrum showed four distinct and well-characterized intense diffraction peaks at scattering angles $(2 \theta)$ of $38.5^{\circ}, 44.6^{\circ}, 64.64^{\circ}$, and $77.45^{\circ}$ corresponding, respectively, to (111), (200), (220), and (311) sets of lattice planes of face-centered cubic (fcc) structure of metallic silver (Fig. 4). The intensity of the diffraction peak corresponding to the (111) plane was more significant than that of the rest of the diffraction peaks in the recorded spectrum, and it can be construed from the above that the (111) plane is the preferred plane of orientation for the silver nanoparticle crystal lattice. The TEM images recorded on the samples further corroborate the crystalline nature of the synthesized AgNPs. The Scherrer formula was used to calculate the average size of the synthesized AgNPs employing the width of the peak, as a representative one, corresponding to the (111) diffraction plane with an applied geometric 


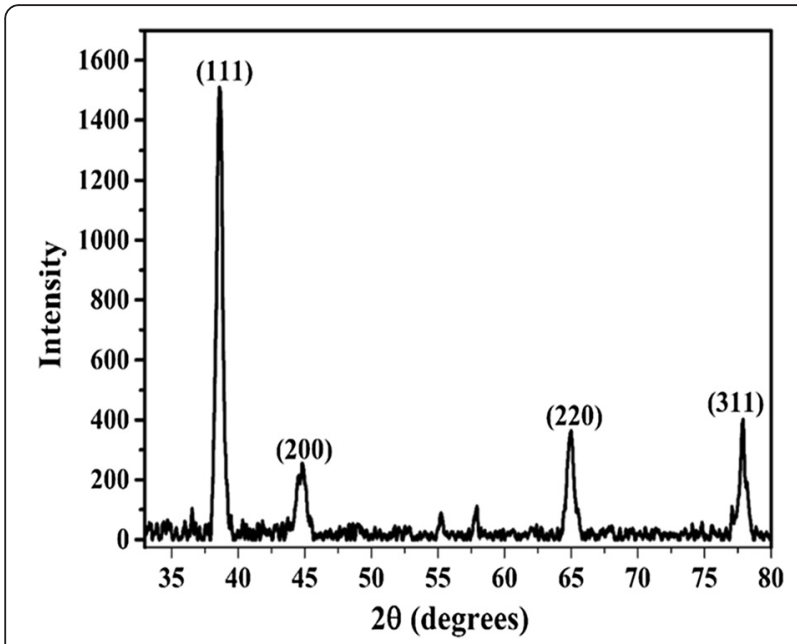

Fig. 4 X-ray diffraction pattern of GT-capped AgNPs in an aqueous system using gum tragacanth as reducing and stabilizing agent, showing face-centered cubic (fcc) crystal structure

factor of 0.97 (Kora and Arunachalam 2012). The resultant average particle size was found to be about $17.5 \mathrm{~nm}$, and the same was found to be in agreement with the size obtained from the corresponding TEM image of AgNPs.

\section{TEM analysis}

The typical TEM images of GT-capped AgNPs synthesized with a composition of $0.5 \%(w / v)$ aqueous $\mathrm{AgNO}_{3}$ solution and $0.5 \%(w / v)$ GT sonicated at $45{ }^{\circ} \mathrm{C}$ for 45 min were depicted in Fig. 5. It was evident from the TEM images that the AgNPs so formed are spherical in shape and were well distributed in the polymer matrix of the gum. The histograms plotted on the obtained data (Fig. 6) to study the particle size distribution reveals that the size of the nanoparticles ranged from 10 to $29 \mathrm{~nm}$ and the average particle size was about $18 \pm 2.0 \mathrm{~nm}$. The selected area electron diffraction (SAED) pattern of the

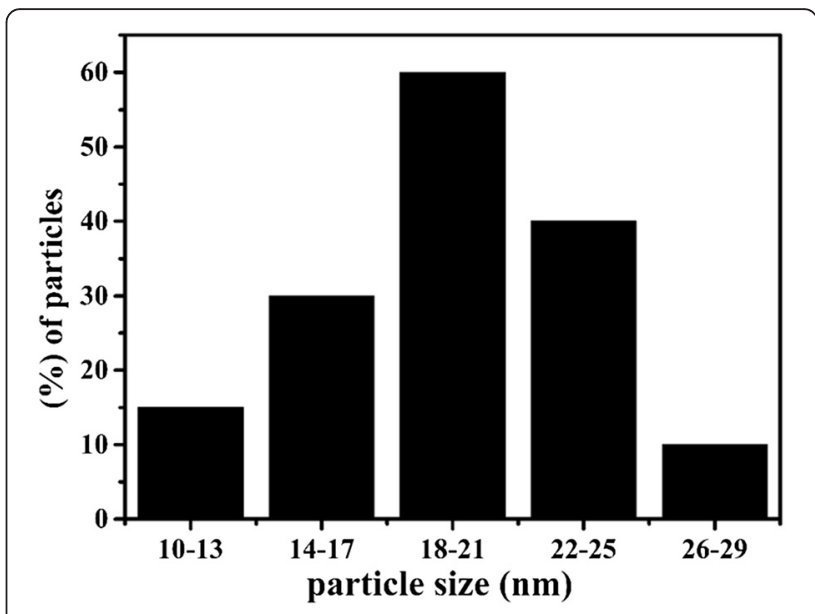

Fig. 6 Histogram showing particle size distribution of GT-capped AgNPs

synthesized AgNPs (Fig. 7) shows concentric rings dotted with bright spots. This indicates the highly crystalline nature of these nanoparticles, and these concentric rings can be attributed to the diffraction from the (111), (200), (220), and (311) planes of the fcc structure of the metallic silver. Further, the stability of the aqueous solution of AgNPs was assessed by zeta potential analysis. A zeta potential value of $-11.21 \mathrm{mV}$ (Fig. 8) indicates that the dispersed AgNPs were capped by negatively charged groups proving that they are stable.

\section{Catalytic activity of AgNPs}

The catalytic ability of AgNPs was studied as it is a very well-known fact that some of the reactions are, otherwise, difficult to take place. Here, the catalytic reduction of $\mathrm{MB}$ and $\mathrm{CR}$ was investigated by employing $\mathrm{NaBH}_{4}$ as the reducing agent and GT-capped AgNPs as the catalyst. It is evident from the previous studies that the efficacy of the metal nanoparticles as electron transfer

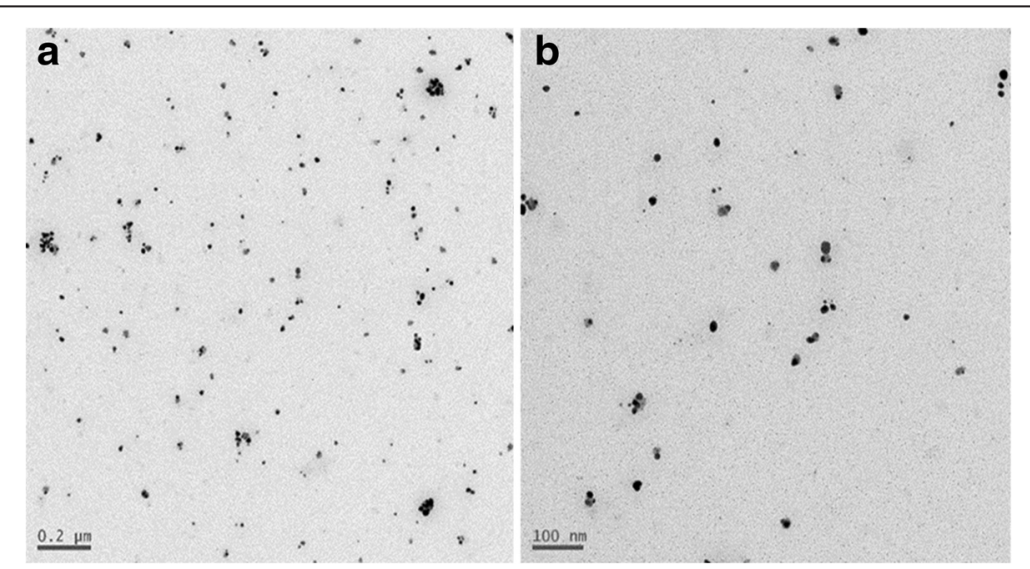

Fig. 5 Typical TEM image of GT-capped AgNPs in an aqueous system using gum tragacanth as reducing and stabilizing agent $\mathbf{a}$ at $0.2-\mu m$ scale and (b) at 100-nm scale 


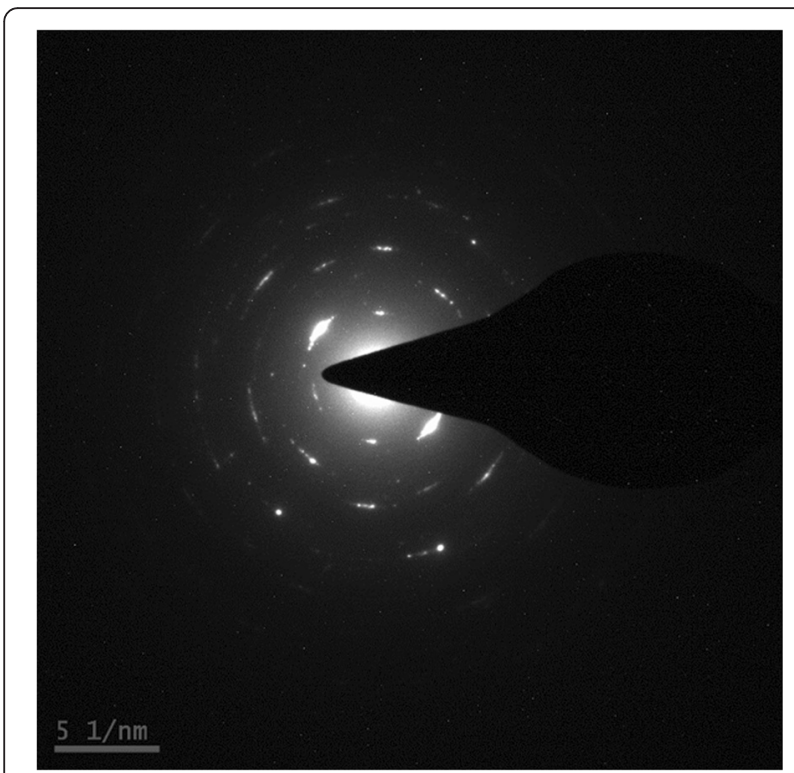

Fig. 7 The SAED pattern of the GT-capped AgNPS

catalysts greatly varies with the size of the metal nanoparticles. The UV-Vis spectra recorded for the reduction of $\mathrm{MB}$ and $\mathrm{CR}$ by $\mathrm{NaBH}_{4}$ in the presence and the absence of AgNPs as catalyst were shown in Fig. 9a-f. Figure 9a depicts the UV-Vis spectrum of reduction of $\mathrm{MB}$ by $\mathrm{NaBH}_{4}$ in the absence of nanocatalysts recorded in 10 -min intervals for a period of $100 \mathrm{~min}$ at room temperature. Figure $9 \mathrm{~b}$ shows the UV-Vis spectrum for the catalytic reduction of $\mathrm{MB}$ by $\mathrm{NaBH}_{4}$ in the presence of silver nanoparticles in 1-min intervals for a period of $12 \mathrm{~min}$ at $25{ }^{\circ} \mathrm{C}$. Similarly, Fig. 9d depicts the UV-Vis spectrum of reduction of $\mathrm{CR}$ by $\mathrm{NaBH}_{4}$ in the absence of nanocatalysts recorded in 10-min intervals for a period of $120 \mathrm{~min}$ at $25{ }^{\circ} \mathrm{C}$, and Fig. 9e shows the absorption spectrum of the nanocatalyst, AgNP-assisted reduction of $\mathrm{CR}$ by $\mathrm{NaBH}_{4}$ in 1-min intervals for a period of $15 \mathrm{~min}$, at $25{ }^{\circ} \mathrm{C}$. The aqueous reaction mixture of $\mathrm{MB}$ and $\mathrm{NaBH}_{4}$ showed a maximum absorption band $\left(\lambda_{\max }\right)$ at $664 \mathrm{~nm}$ with a shoulder at $614 \mathrm{~nm}$ whereas, in case of the $\mathrm{CR}$ and $\mathrm{NaBH}_{4}$ system, the $\lambda_{\max }$ appeared at $500 \mathrm{~nm}$ with a shoulder at $350 \mathrm{~nm}$. The absorption intensity variation at 664 and $500 \mathrm{~nm}$ for $\mathrm{MB}$ and $\mathrm{CR}$, respectively, signifies the rapid reaction rate over a measured period of time. It was observed that the rate of reaction, in both the cases of $M B$ and $C R$, increased with the increase in reaction time, and the reduction of $\mathrm{MB}$ and $\mathrm{CR}$ is near complete at the end of the reaction period, signifying effectiveness of the nanocatalyst. On the contrary, the rate of reaction in the event of the absence of the nanocatalyst in reducing $\mathrm{MB}$ and $\mathrm{CR}$ was very slow, and it was observed that the reaction has never been complete. The quantitative estimation of reduction of both the dyes, i.e., $\mathrm{MB}$ and $\mathrm{CR}$, can be monitored from Fig. 9b, e, respectively, indicating the complete catalytic reduction of both the dyes with GTcapped AgNPs as nanocatalyst. The molecular structures of $\mathrm{MB}$ and $\mathrm{CR}$ and possible reduction products were shown in Fig. 10a, b, respectively.

In the reaction mixture, a higher concentration of $\mathrm{NaBH}_{4}$ was employed as compared to that of both $\mathrm{MB}$ and $\mathrm{CR}$, which results in the rise of the $\mathrm{pH}$ of the whole system. This, in turn, results in retarding the degradation of $\mathrm{BH}_{4}^{-}$ions. The liberated $\mathrm{H}^{+}$ions create a purging atmosphere over the reaction mixture thereby effectively inhibiting the aerial oxidation of reduced $\mathrm{MB}$ and $C R$ products. The catalytic reduction aided by metallic nanoparticles is being effectively carried out by transferring electrons from electron donor species $\mathrm{BH}_{4}^{-}$ to electron acceptor species $\mathrm{MB}$ and $\mathrm{CR}$ mediated by GT-capped AgNPs and thus reduces the activation energy and contributes to the stabilization of the system. As the $\mathrm{NaBH}_{4}$ concentration in the system is much higher as compared to that of $\mathrm{MB}$ and $\mathrm{CR}$, the reaction rate can be assumed to be independent of the $\mathrm{NaBH}_{4}$ concentration, and hence, a pseudo-first-order kinetics could be used to evaluate the kinetic reaction rates of the present catalytic reduction systems. A linear correlation

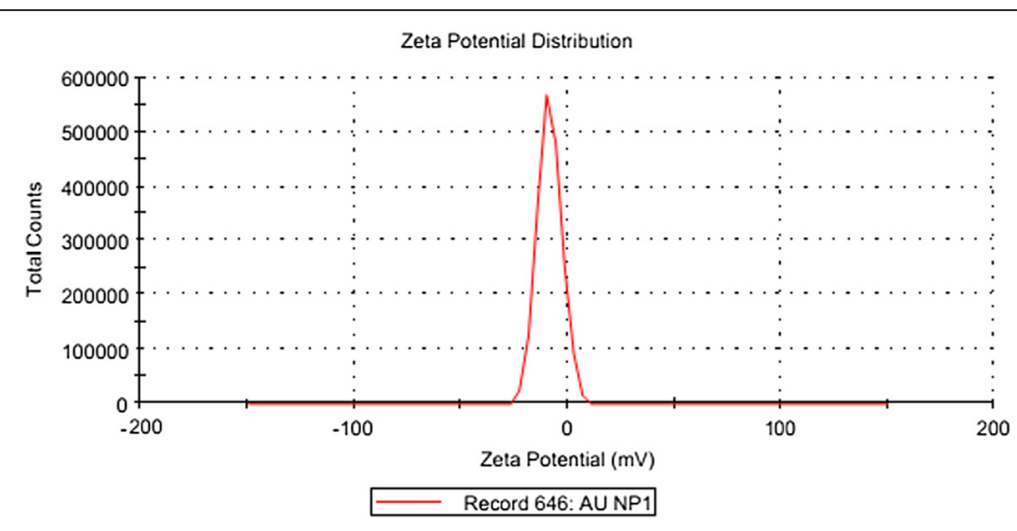

Fig. 8 Graph of zeta potential analysis for aqueous AgNPs 

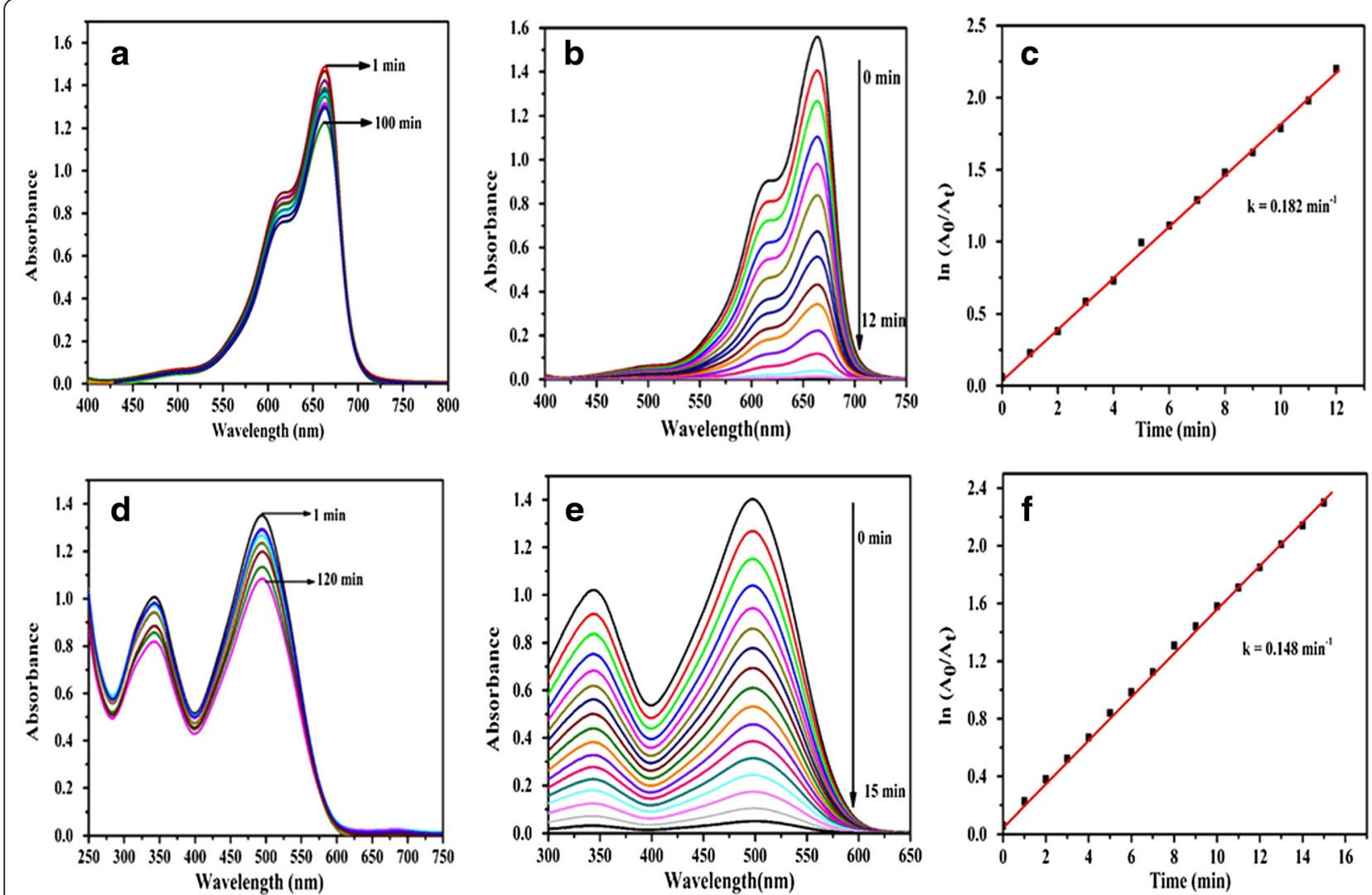

Fig. 9 a UV-Vis spectrum of reduction of $\mathrm{MB}$ by $\mathrm{NaBH}_{4}$ in the absence of nanocatalysts recorded in 10-min intervals for a period of $100 \mathrm{~min}$ at room temperature. $\mathbf{b} \mathrm{UV}$-Vis spectrum for the catalytic reduction of $\mathrm{MB}$ by $\mathrm{NaBH}_{4}$ in presence of silver nanocolloids in 1-min intervals for a period of $12 \mathrm{~min}$ at room temperature. c Plot of $\ln \left(A_{0} / A_{t}\right)$ against time for pseudo-first-order reaction for the catalytic reduction of $\mathrm{MB}$ with excess $\mathrm{NaBH}_{4}$ in the presence of GT-capped AgNPs. $\mathbf{d}$ UV-Vis spectrum of reduction of CR by $\mathrm{NaBH}_{4}$ in the absence of nanocatalysts recorded in 10-min intervals for a period of 120 min at $25^{\circ} \mathrm{C}$. e UV-Vis absorption spectrum of nanocatalyst (AgNPs)-assisted reduction of $\mathrm{CR}_{\text {by }} \mathrm{NaBH}_{4}$ in 1-min intervals for a period of 15-min room temperature. $\mathbf{f}$ Plot of $\ln \left(A_{0} / A_{t}\right)$ against time for pseudo-first-order reaction for the catalytic reduction of $\mathrm{MB}$ with excess $\mathrm{NaBH}_{4}$ in the presence of GT-capped AgNPs

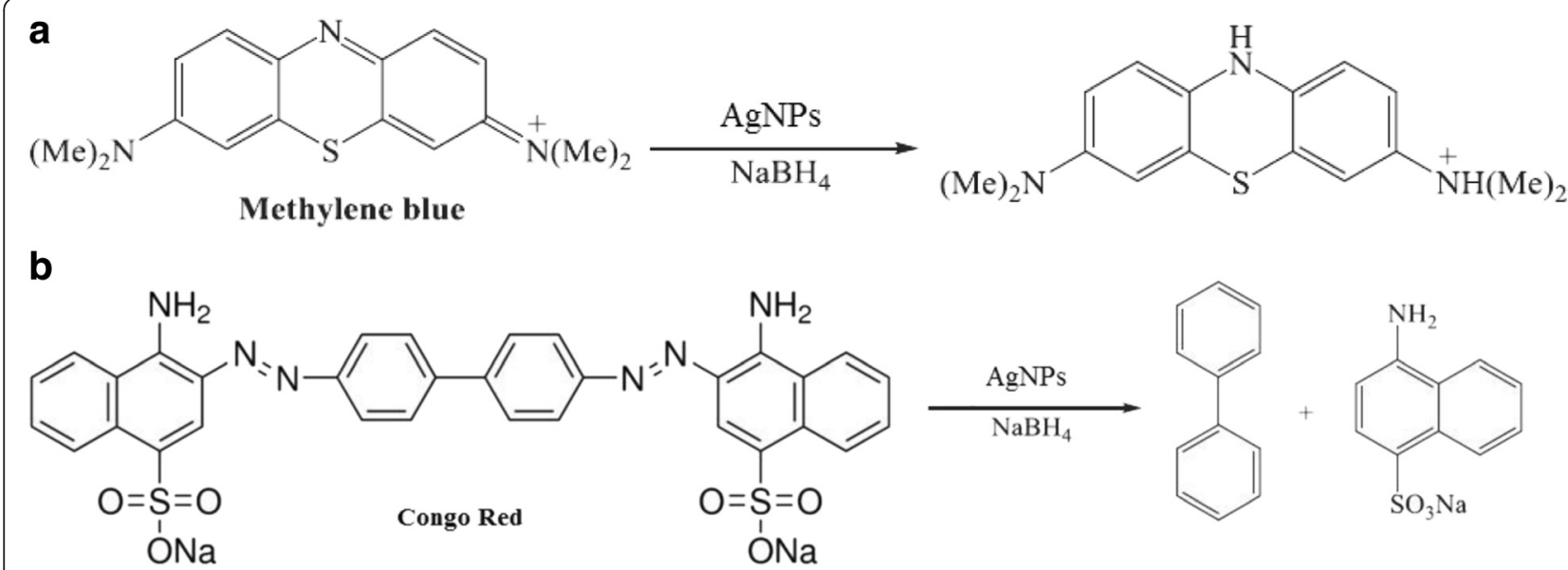

Fig. 10 a Structure and reduction products of MB with GT-capped AgNPs and $\mathbf{b}$ structure and reduction products of CR with GT-capped AgNPs 
between $\ln \left(A_{0} / A_{t}\right)$ (where $A_{0}$ is the absorbance at the initial time, $A_{t}$ is the absorbance at the specific reaction time) at the room temperature establishes that the reaction is of pseudo-first order. The pseudo-first-order rate constants $(k)$ for the reduction reactions of $\mathrm{MB}$ (Fig. 9c) and CR (Fig. 9f) calculated from the slope are 0.182 and $0.148 \mathrm{~min}^{-1}$, respectively. The catalyst turnover number (TON) and the turnover frequency (TOF) are two important parameters used to determine the catalyst efficiency. In heterogeneous catalysis, the TON is the number of reactant molecules that $1 \mathrm{~g}$ of catalyst can convert into products (Lin et al. 2013). The TOF is simply TON/time. For MB and CR dyes using AgNPs as catalysts at a rate of $1.1 \mathrm{~g} / \mathrm{L}$ dose, the TOF was found to be $2.6 \times 10^{16}$ and $2.1 \times 10^{16}$ molecules/g/s, respectively.

\section{Conclusions}

In this paper, we report a simple, eco-friendly green synthesis of AgNPs employing naturally occurring and renewable GT as a reducing and stabilizing agent as against using harsher and toxic chemical entities. The nanoparticles so produced here are highly stable as well as reactive due to reactive functional groups present on the attached natural gum entity. The utilization of these green synthesized AgNPs in a plethora of crucial applications such as, among one of many, the catalytic reduction of carcinogenic dyes like MB and CR was reported. As we can see, the greener synthesis of AgNPs, by using exudates from the renewable plant resources, follows the very tenets of green chemistry, and hence, the process can be dubbed as eco-friendly in the true sense. It was very much evident from the experimental results that the catalytic reduction of $\mathrm{MB}$ and $\mathrm{CR}$ in the presence of $\mathrm{NaBH}_{4}$ with AgNPs as a catalyst was near complete indicating the effectiveness of the nanocatalyst used. The high degree of catalytic ability of GT-capped AgNPs in accelerating the catalytic reduction certainly draws greater importance due to the cleaner and greener approach.

\begin{abstract}
Abbreviations
AgNPs, silver nanoparticles; $\mathrm{cm}$, centimeter; $\mathrm{CR}$, Congo red; FTIR, Fourier transform infrared; g, gram; GT, gum tragacanth; $M B$, methylene blue; min, minute; $\mathrm{mL}$, milliliter; $\mathrm{nm}$, nanometer; rpm, revolutions per minute; TEM, transmission electron microscopy; $w / v$, weight/volume; XRD, X-ray diffraction
\end{abstract}

\section{Authors' contributions}

IMK, GBR, and DRK synthesized the AgNPs, BRK collected gum tragacanth and prepared the aqueous gum extract, and DRK characterized the AgNPs using UV-Vis spectroscopy. GBR characterized the AgNPs using X-ray diffraction and TEM. IMK conducted catalytic degradation studies on AgNPs. GVB contributed in analysing the data. All authors read and approved the final manuscript.

\section{Competing interests}

The authors declare that they have no competing interests.

Received: 14 March 2016 Accepted: 26 July 2016

Published online: 13 August 2016

\section{References}

Callegari A, Tonti D, Chergui M. Photochemically grown silver nanoparticles with wavelength-controlled size and shape. Nano Lett. 2003;3:1565-8.

Chenlo F, Moreira R, Silva C. Rheological behaviour of aqueous systems of tragacanth and guar gums with storage time. J Food Eng. 2010;96:107-13. doi:10.1016/j.jfoodeng.2009.07.003.

Daniel M, Astruc D. Gold nanoparticles: assembly, supramolecular chemistry, quantum-size-related properties, and applications toward biology, catalysis, and nanotechnology. Chem Rev. 2004;104:293-346.

Firdhouse MJ, Lalitha P, Sripathi SK. Novel synthesis of silver nanoparticles using leaf ethanol extract of Pisonia grandis (R. Br). Der Pharma Chem. 2012;4:2320-6.

Ganapuram BR, Alle M, Dadigala R, et al. Catalytic reduction of methylene blue and Congo red dyes using green synthesized gold nanoparticles capped by salmalia malabarica gum. Int Nano Lett. 2015;5:215-22. doi:10.1007/s40089-015-0158-3.

Guo S, Wang E. Synthesis and electrochemical applications of gold nanoparticles. Anal Chim Acta. 2007;598:181-92. doi:10.1016/j.aca.2007.07.054.

Hassan AG, Dalgaard MJ, Anne MS (2013) Tragacanth gum: structural composition, natural functionality, and enzymatic conversion as source of potential prebiotic activity. Thesis, Technical University of Denmark

Junejo Y, Sirajuddin S, Baykal A, et al. A novel green synthesis and characterization of Ag NPs with its ultra-rapid catalytic reduction of methyl green dye. Appl Surf Sci. 2014;290:499-503. doi:10.1016/j.apsusc.2013.11.106

Karcher S, Kornmüller A, Jekel M. Anion exchange resins for removal of reactive dyes from textile wastewaters. Water Res. 2002;36(19):4717-24.

Kokila T, Ramesh PS, Geetha D. Biosynthesis of silver nanoparticles from Cavendish banana peel extract and its antibacterial and free radical scavenging assay: a novel biological approach. Appl Nanosci. 2015;5:911-20. doi:10.1007/s13204-015-0401-2.

Kora AJ, Arunachalam J. Green fabrication of silver nanoparticles by gum tragacanth (Astragalus gummifer): a dual functional reductant and stabilizer. J Nanomater. 2012;2012:1-8. doi:10.1155/2012/869765.

Kora AJ, Beedu SR, Jayaraman A. Size-controlled green synthesis of silver nanoparticles mediated by gum ghatti (Anogeissus latifolia) and its biological activity. Organic Med Chem Lett. 2012;2:17. doi:10.1186/2191-2858-2-17.

Li T, Park HG, Choi S-H. Y-Irradiation-induced preparation of Ag and Au nanoparticles and their characterizations. Mater Chem Phys. 2007;105:325-30. doi:10.1016/j.matchemphys.2007.04.069.

Lin C, Tao K, Hua D, Ma Z, Zhou S. Size effect of gold nanoparticles in catalytic reduction of p-nitrophenol with $\mathrm{NaBH}_{4}$. Molecules. 2013;18:12609-20. doi:10.3390/molecules181012609.

Maciollek A, Ritter $\mathrm{H}$. One pot synthesis of silver nanoparticles using a cyclodextrin containing polymer as reductant and stabilizer. Beilstein J Nanotechnol. 2014;5:380-5. doi:10.3762/bjnano.5.44.

Mehndiratta P, Jain A, Srivastava S, Gupta N. Environmental pollution and nanotechnology. Environ Pollut. 2013;2:49-58. doi:10.5539/ep.v2n2p49.

Murali Krishna I, Bhagavanth Reddy G, Veerabhadram G, Madhusudhan A. Ecofriendly green synthesis of silver nanoparticles using salmalia malabarica: synthesis, characterization, antimicrobial, and catalytic activity studies. Appl Nanosci. 2015. doi:10.1007/s13204-015-0479-6.

Murugadoss A, Chattopadhyay A. A "green" chitosan-silver nanoparticle composite as a heterogeneous as well as micro-heterogeneous catalyst. Nanotechnology. 2008;19:015603. doi:10.1088/0957-4484/19/01/015603.

Pandey S, Mishra SB. Sol-gel derived organic-inorganic hybrid materials: synthesis, characterizations and applications. J Sol-Gel Sci Technol. 2011;59(1):73-94

Pandey S, Mishra SB. Green synthesis of polysaccharide/gold nanoparticle nanocomposite: an efficient ammonia sensor. Corbopol. 2013;94:229-34.

Pandey S, Mishra SB. Catalytic reduction of $p$-nitrophenol by using platinum nanoparticles stabilised by guar gum. Corbopol. 2014;113:525-31. doi:10.1016/j.carbpol.2014.07.047.

Pandey S, Mishra SB. Au nanocomposite based chemiresistive ammonia sensor for health monitoring. Am Chem Soc. 2016;1(1):55-62. doi:10.1021/ acssensors.5b00013.

Pandey S, Goswami GK, Nanda KK. Green synthesis of biopolymer-silver nanoparticle nanocomposite: an optical sensor for ammonia detection. Int J Biological Macromolecules. 2012;51(4):583-9.

Pandey S, Goswami GK, Nanda KK. Nanocomposite based flexible ultrasensitive resistive gas sensor for chemical reactions studies. Sci Rep. 2013;3:2082. doi:10.1038/srep02082. 
Pandey S, Ramontja J. Turning to nanotechnology for water pollution control: applications of nanocomposites, Focus on Sciences. 2016. doi:10.20286/ focsci-020219.

Parveen M, Ahmad F, Malla AM, Azaz S. Microwave-assisted green synthesis of silver nanoparticles from Fraxinus excelsior leaf extract and its antioxidant assay. Appl Nanosci. 2016;6:267-76. doi:10.1007/s13204-015-0433-7.

Pyatenko A, Shimokawa K, Yamaguchi M, et al. Synthesis of silver nanoparticles by laser ablation in pure water. Appl Phys A. 2004;79:803-6. doi:10.1007/ s00339-004-2841-5.

Rai M, Yadav A, Gade A. Silver nanoparticles as a new generation of antimicrobials. Biotechnol Adv. 2009;27:76-83. doi:10.1016/j.biotechadv. 2008.09.002.

Raju D, Paneliya N, Mehta UJ. Extracellular synthesis of silver nanoparticles using living peanut seedling. Appl Nanosci. 2014;4:875-9. doi:10.1007/s13204-013-0269-y.

Rastogi L, Sashidhar RB, Karunasagar D, Arunachalam J. Gum kondagogu reduced/stabilized silver nanoparticles as direct colorimetric sensor for the sensitive detection of $\mathrm{Hg}^{2+}$ in aqueous system. Talanta. 2014;118:111-7. doi:10.1016/j.talanta.2013.10.012.

Raveendran P, Fu J, Wallen SL. Completely "green" synthesis and stabilization of metal nanoparticles. J Am Chem Soc. 2003;125(46):13940-1.

Roy K, Sarkar CK, Ghosh CK. Plant-mediated synthesis of silver nanoparticles using parsley (Petroselinum crispum) leaf extract: spectral analysis of the particles and antibacterial study. Appl Nanosci. 2015;5:945-51. doi:10.1007/s13204-014-0393-3.

Solomon SD, Bahadory M, Jeyarajasingam AV, et al. Synthesis and study of silver nanoparticles. J Chem Educ. 2007;84:322-5.

Song JY, Kim BS. Rapid biological synthesis of silver nanoparticles using plant leaf extracts. Bioprocess Biosyst Eng. 2009;32:79-84. doi:10.1007/s00449-008-0224-6.

Suvith VS, Philip D. Catalytic degradation of methylene blue using biosynthesized gold and silver nanoparticles. Spectrochim Acta - Part A Mol Biomol Spectrosc. 2014;118:526-32. doi:10.1016/j.saa.2013.09.016.

Thakkar KN, Mhatre SS, Parikh RY. Biological synthesis of metallic nanoparticles. Nanomedicine. 2010;6:257-62. doi:10.1016/j.nano.2009.07.002.

Tischer CA, lacomini M, Gorin PAJ. Structure of the arabinogalactan from gum tragacanth (Astralagus gummifer). Carbohydr Res. 2002;337:1647-55.

Wang LS, Wang CY, Yang CH, Hsieh CL, Chen SY, Shen CY, Wang JJ, Huang KS. Synthesis and anti-fungal effect of silver nanoparticles-chitosan composite particles. Int J Nanomed. 2015:10:2685-96.

Wanyonyi WC, Onyari JM, Shiundu PM. Adsorption of Congo red dye from aqueous solutions using roots of Eichhornia crassipes: kinetic and equilibrium studies. Energy Procedia. 2014;50:862-9. doi:10.1016/j.egypro.2014.06.105.

Zalikha N, Azlina W, Ab W, et al. Adsorption of methylene blue by agricultural solid waste of pyrolyzed EFB biochar. J Purity, Util React Environ. 2012;1:376-90.

\section{Submit your manuscript to a SpringerOpen ${ }^{\circ}$ journal and benefit from:}

- Convenient online submission

- Rigorous peer review

- Immediate publication on acceptance

- Open access: articles freely available online

- High visibility within the field

- Retaining the copyright to your article

Submit your next manuscript at $>$ springeropen.com 